\title{
A MEDICAÇÃO PRESCRITA NA INTERNAÇÃO HOSPITALAR: o conhecimento do cliente
}

\author{
Ana Carolina Guidorizzi Zanett \\ Sílvia Helena De Bortoli Cassiani \\ Izabela Ramos Moreira Afonso \\ Claudia Câmara Freire \\ Paulo Celso Prado Teles Filho
}

\section{Resumo}

Erros no processo de administração de medicamentos são preocupações de enfermeiros e a sua ocorrência pode ser minimizada com a orientação do cliente quanto à terapia medicamentosa. Este estudo descritivo, teve como objetivo identificar o grau de informação de clientes hospitalizados, acerca dos medicamentos que Ihes são prescritos. A metodologia utilizada foi a exploratória-descritiva. Foram entrevistados 76 clientes adultos, hospitalizados, entre 18 a 60 anos, sendo $69,7 \%$ do sexo feminino. Destacou-se dentre outros, que $28 \%$ não apresentavam conhecimento acerca do nome do primeiro medicamento prescrito e $55 \%$ não souberam referir a dosagem. Detectou-se um número razoável de clientes que desconheciam a medicação prescrita ou tinham conhecimento incorreto. Faz-se necessário a conscientização dos profissionais de enfermagem quanto à importância da orientação ao cliente acerca dos medicamentos durante a internação.

Descritores: enfermagem; erros: preparações farmacológicas

\begin{abstract}
Errors along the process of medicine administration are some concerns referred to by nurses, and their occurrence can be minimized by providing clients with some guidance regarding medicine-based therapy. This descriptive study aimed at identifying inpatients level of information concerning medication prescribed to them. Exploratory and descriptive methodology was used. Seventy-six adult inpatients were interviewed. Their age ranged from 18 to 60 years, and $69.7 \%$ of them were female. Twenty-eight percent did not know the name of the first medicine prescribed to them, and 55\% were not able to give information about their dosage. A reasonable number of clients knew nothing about medication they were using, or had misconceptions about it. A thorough understanding is paramount to Nursing professionals on their role to provide their patients with information on medication they are taking during their hospitalization time.

Descriptors: nursing; errors; pharmacological preparations

Title: Medication Prescribed to Inpatients: The Client's Knowledge
\end{abstract}

\section{Resumen}

Errores en el proceso de administración de medicamentos son preocupaciones de los enfermeros y su ocurrencia se puede minimizar con la orientación del cliente respecto a la terapia medicamentosa. Este estudio descriptivo tuvo como objetivo identificar el grado de información de clientes hospitalizados sobre los medicamentos que les han sido prescriptos. La metodología utilizada fue la exploratoria-descriptiva. Entrevistamos 76 clientes adultos, hospitalizados entre 18 y 60 años, siendo $69.7 \%$ del sexo femenino. $28 \%$ no sabían el nombre del primer medicamento prescripto $y$ $55 \%$ no sabian referir el dosaje. Se detectó un número razonable de clientes que desconocía la medicación prescripta o la sabía incorrectamente. Se hace necesaria la concienciación de los profesionales de enfermería respecto a la importancia de orientar al cliente para que no se cometan errores durante la internación.

Descriptores: enfermería; errores; preparaciones farmacológicas

Título: La Medicación Prescripta En La Hospitalización: El Conocimiento Del Cliente

\section{Introdução}

A equipe de enfermagem junto a outros profissionais de saúde, tais como, médicos e farmacêuticos, são os principais responsáveis pelas atividades terapêuticas ligadas à administração de medicamentos. É responsabilidade da equipe de enfermagem compreender os efeitos da droga, administrála corretamente e monitorar as respostas do cliente(1). A administração de medicamentos, por se tratar de uma atividade diária da enfermagem, requer experiência e exige responsabilidades ${ }^{(2)}$.

Para sua execução, é necessária a aplicação de vários princípios científicos que fundamentam a ação dos profissionais, promovendo a segurança do paciente e do próprio profissional. O enfermeiro, para administrar medicamentos com segurança, eficiência e responsabilidade, deve conhecer o paciente, compreender os efeitos e ações dos medicamentos, administrá-lo corretamente e avaliar a resposta do paciente (3).

A administração de medicamentos é um processo multidisciplinar e um multissistema iniciando no momento da prescrição médica, continuando com a provisão deste medicamento pelo farmacêutico e terminando com a sua preparação e administração aos clientes ${ }^{(4)}$. No Brasil cabe aos enfermeiros a supervisão de auxiliares e técnicos de enfermagem durante a administração de medicamentos. No entanto, foi constatado que esta supervisão nem sempre é realizada, podendo levar a erros de medicação a qualquer momento.

Esses erros são motivos de preocupação no trabalho dos enfermeiros e um dos indicadores da qualidade da assistência de saúde prestada aos pacientes hospitalizados ${ }^{(5)}$. O aumento da incidência de erros de medicação nos hospitais e principalmente a subnotificação são preocupações de todos os profissionais. Intervenções devem ser empreendidas para minimizar essa situação. A ocorrência de erros pode trazer sérias conseqüências e até o óbito dos clientes ${ }^{(6)}$.

Erros de medicação são incidentes associados com os medicamentos ocorridos durante a prescrição, transcrição, distribuição e administração. São classificados em dois tipos erros de omissão e erros de condução(7). Esses incidentes, sempre envolvem um enfermeiro ou equipe em associação com médico, farmacêutico, provedores e a instituição de cuidado. Para evitá-los, tem-se de utilizar os cinco certos na administração de medicamentos, os quais são as bases da educação no ensino de administração de medicamentos em escolas de enfermagem. Os cinco certos advertem o enfermeiro quanto a administração do medicamento certo, para o paciente certo, com a dose certa, na via certa e no horário certo. Além disso, a instituição do sistema de dose unitária e preparação

\footnotetext{
* Enfermeiras graduadas pela Escola de Enfermagem de Ribeirão Preto - Universidade de São Paulo - EERP/USP.

** Enfermeira. Professora Associada junto ao Departamento de Enfermagem Geral e Especializada da EERP/USP.

*** Enfermeiro. Doutorando da EERP/USP. Docente e Chefe do Departamento de Pesquisa da UNICASTELO - Campus VIII - Descalvado - SP. E-mail do autor: shbcassi@eerp.usp.br
} 
de endovenosos por farmácia pode diminuir a incidência dos erros de medicação, além de proporcionar à enfermagem um tempo extra para o cuidado do cliente $^{(8)}$.

O conhecimento do cliente sobre sua medicação, através da orientação fornecida por enfermeiros, vem sendo apresentado como uma das soluções para que os erros possam ser minimizados. O cliente bem informado pode evitar danos a si próprio durante a administração de seus medicamentos. A educação é obviamente uma importante parte na prevenção dos erros de medicação.

Assim, o paciente bem informado pode ajudar na prevenção de erros na medicação e outros eventos adversos, o que depende do fornecimento de informações fornecidas pelos enfermeiros, médicos e farmacêuticos ${ }^{(9)}$. Alguns incidentes nos quais o paciente ou seus familiares são suficientemente informados, podem ser prevenidos.

Os enfermeiros devem dar ênfase na educação do paciente, pois ensiná-los é um componente essencial para o seu cuidado. Além disso, os enfermeiros têm mais oportunidades de ensinar e educar os pacientes em relação aos outros membros da saúde. Algumas das melhorias que também podem ser feitas incluem a estruturação de programas de aprendizado, redação de informações, realização de esquemas de auto-medicação, aconselhamento e aumento da comunicação com os profissionais da área farmacêutica. Diante dos aspectos apontados, este estudo teve o propósito de identificar o grau de informação de clientes hospitalizados acerca dos medicamentos que lhe são prescritos.

\section{Objetivo}

O objetivo deste estudo foi identificar o grau de informação de clientes hospitalizados, acerca dos medicamentos que the são prescritos. O grau de informação pretendido refere-se ao conhecimento do nome do medicamento, dose, via de administração, horário e propósito.

\section{Material e método}

Trata-se de um estudo descritivo, com amostra conveniente de 76 clientes adultos, de ambos os sexos, internados em um hospital universitário do interior do estado de São Paulo, em dezembro de 2000. Foram excluídos os clientes das clínicas psiquiátrica e UTI e os que não foram capazes de prestar informações verbais. Para a coleta de dados, utilizou-se a técnica de entrevista estruturada através de um questionário e do registro de dados da prescrição médica. O roteiro de entrevista foi desenvolvido e avaliado por juízes no que refere ao conteúdo e consistia de questões sobre a identificação, nome, via de administração, horário, propósito e dose do medicamento prescrito.

Após o projeto ter sido aprovado pelo Comitê de Ética em Pesquisa da instituição hospitalar em estudo, bem como após a obtenção do consentimento informado dos clientes, a coleta de dados foi iniciada. Foram entrevistados os clientes adultos, conscientes e hospitalizados durante a visita da primeira pesquisadora na clínica. A seguir os dados foram organizados para análise, utilizando-se o programa Epiinfo e apresentados de forma descritiva, utilizando tabelas e quadros.

\section{Resultados e discussão}

Dos $76(100 \%)$ clientes, 31,6\% encontrava-se na faixa etária de 30 a 39 anos e $69,7 \%$ eram do sexo feminino. Dos sujeitos estudados, $46,1 \%$ tinham o primeiro grau incompleto.

Em relação à internação: $45(59,2 \%)$ dos clientes foram internados apenas uma vez em hospitais, no ano de 2000. Dos clientes estudados, $56(73,7 \%)$ foram internados pelo menos uma vez no hospital em estudo. No que refere à via de administração de medicamentos, a maioria $63,2 \%$ dos sujeitos a recebiam por via oral, 5,3\% por via intramuscular, $2,6 \%$ por via subcutânea e $28,9 \%$ por via endovenosa.

Ao serem questionados sobre o medicamento que recebiam, $55(72,4 \%)$ informaram corretamente o nome do primeiro medicamento prescrito, 23,6\% acertaram parcialmente ou errou e 3,9\% não citaram.

Em relação a via de administração de medicamentos 68 $(89,5 \%)$ dos clientes referiram corretamente a via de administração do primeiro medicamento prescrito, 6,6\% acertaram parcialmente ou erraram e 3,9\% não citaram.

Quanto ao horário do primeiro medicamento prescrito $48(63,2 \%)$ dos clientes responderam corretamente, 32,9\% acertaram parcialmente ou erraram e 3,9\% não citaram.

Ao analisar os dados obtidos constatou-se que o conhecimento dos clientes em relação ao nome, via de administração e horário do primeiro medicamento prescrito, mostrou-se satisfatório. Esse dado parece apontar um avanço nas medidas de educação ao cliente hospitalizado, ou seja, o cliente informado é capaz de evitar a ocorrência de possíveis erros de medicação durante a administração de medicamentos e, nesse hospital, os clientes, em sua maioria tem conhecimento das medicações. O uso incorreto de medicações é uma das causas das reações das drogas e a orientação pode aumentar o conhecimento da terapêutica medicamentosa, desse modo, diminuindo os problemas advindos dessa prática ${ }^{(10)}$.

Enfermeiros são preparados para realizar a ponte clienteeducação, pois, a educação do cliente vem sendo uma tradicional atividade da prática de enfermagem. Educar clientes sobre medicação requer uma parcela de cuidado e planejamento(11). $^{(1)}$.

Do total de entrevistados, $59(77,6 \%)$ informaram corretamente o propósito do primeiro medicamento prescrito. Em relação a dose 19 (25\%) responderam corretamente a dose do primeiro medicamento prescrito, o que pode ser observado no gráfico 1.

\section{GRÁFICO 1-DISTRIBUIÇÃO NUMÉRICA DOS CLIENTES HOSPITALIZADOS SEGUNDO O CONHECIMENTO DA DOSAGEM DO} PRIMEIRO MEDICAMENTO PRESCRITO. RIBEIRÃO PRETO, 2001.

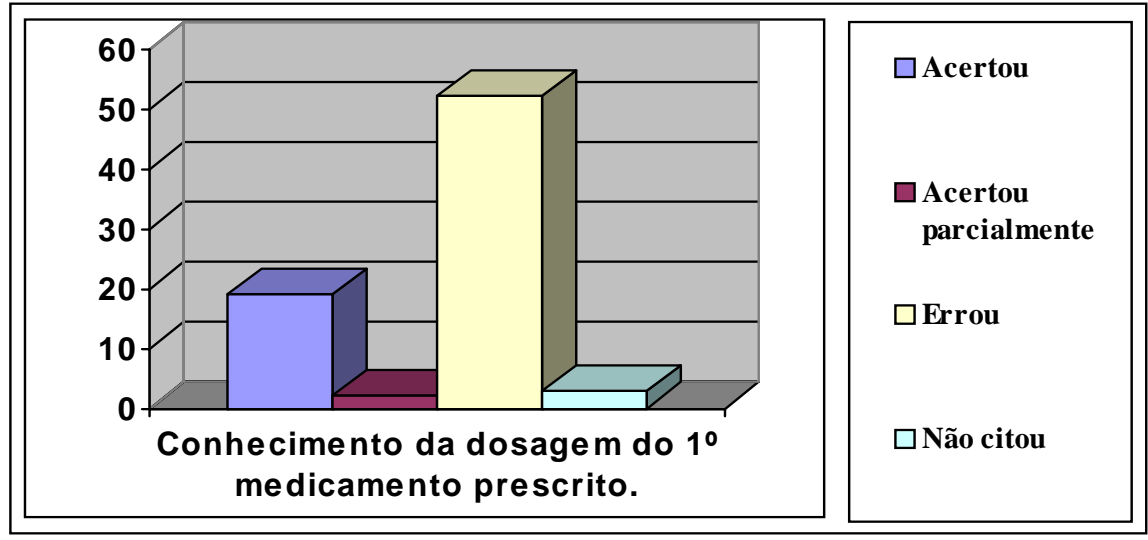


A análise dos resultados mostrou que a maior parte 52 $(68,4 \%)$ dos clientes não acertaram a dose do primeiro medicamento prescrito, mostrando a necessidade de implantação de medidas que sejam capazes de fornecer o conhecimento necessário para que o cliente possa tornar-se um parceiro na administração de medicamento. Contribuindo para a redução da ocorrência de erros.

Para educar o cliente em relação à medicação que está recebendo, a equipe de enfermagem deve instruí-lo durante a administração de cada um dos medicamentos, orientando-o sobre o nome, via de administração, dose, propósito e a forma do medicamento. Nos EUA, foi desenvolvido um auxílio para o ensino, o qual apresenta uma lista individual das medicações, com o propósito de cada um, a freqüência de administração e potenciais efeitos adversos ${ }^{(10)}$

Foram instituídas nove regras para a equipe de enfermagem na educação de clientes sobre medicação, as quais se seguem:

1. Encorajar o cliente a manter uma lista escrita das suas medicações. Ela deve incluir os nomes e validade dessas medicações e horário de cada medicação.

2. Reforçar a importância de comunicar os membros da equipe de saúde sobre alergias ou sensibilidades que tiveram com medicamentos passados.

3. Mostrar a necessidade de saber profundamente como os clientes devem tomar suas medicações (à noite, pela manhã, com alimentos, e assim por diante).

4. Encorajar o cliente a ser um participante ativo no seu cuidado. O cliente deve ter certeza que a equipe hospitalar, relatou o nome de alguma nova medicação, seu propósito e possíveis efeitos adversos.

5. Mostrar ao cliente como controlar os dias em que tomará a medicação e as chances de ingerir uma dose ou droga errada.

6. Encorajar o cliente a examinar todas as medicações que the são prescritas.

7. Os clientes têm todo direito de fazer perguntas e obter respostas sobre procedimentos ou sobre o seu tratamento.

8. Não ter pressa para verificar se o cliente entendeu a sua prescrição.

9. Aconselhar o cliente a procurar uma farmácia onde conheça o farmacêutico, para que este possa lhe esclarecer dúvidas se necessário.

O cliente deve ser encorajado a ter mais responsabilidade com seu próprio cuidado e suas medicações. Assim o conhecimento sobre suas medicações pode ser maximizado(12).

Ao relacionar o sexo dos clientes entrevistados com os erros obtidos, observou-se que os clientes do sexo masculino obtiveram maior porcentagem (26\%) de erros do que os clientes do sexo feminino (5,6\%). Ao investigar os sujeitos que recebiam mais de uma medicação verificou-se que do total de entrevistados, 88\% receberam uma segunda medicação, $46,3 \%$ sabiam o nome do segundo medicamento prescrito, $17,9 \%$ sabiam a dose, 76,1\% acertaram a via de administração, 55,2\% acertaram o horário e $67,2 \%$ acertaram o propósito. Dos clientes que receberam mais de duas medicações, 53,8\% não citaram o nome do terceiro medicamento prescrito, $72,2 \%$ o nome do quarto medicamento, $85 \%$ o nome do quinto medicamento, $82,8 \%$ o nome do sexto medicamento, $76,5 \%$ o nome do sétimo medicamento e $86,7 \%$ o nome do oitavo medicamento.

Pode-se identificar que o grau de informação de clientes, durante a internação hospitalar, acerca dos medicamentos que Ihe são prescritos é satisfatório. Faz-se necessária a conscientização dos profissionais de enfermagem em relação à importância da orientação ao cliente para a utilização de medicamentos durante o período de internação, para aumentar ainda mais o referido grau.

\section{Considerações finais}

É sabido que danos iatrogênicos em administração de medicamentos ocorrem freqüentemente em pacientes hospitalizados e, geralmente causam seqüelas ${ }^{(13)}$. Os dados obtidos nesse estudo mostram a importância da adoção de medidas que tornem o cliente conhecedor de sua medicação, possibilitando minimizar a ocorrência de erros durante a administração de medicamentos.

Conclui-se que existe necessidade de implementar um programa de educação junto a clientes hospitalizados acerca do uso de medicação. E para que o programa de educação seja efetivo é importante o empenho da equipe de enfermagem, buscando novas tecnologias de ensino que auxiliem os clientes a buscar informação acerca do seu cuidado à saúde, em particular, no que tange aos medicamentos a serem administrados, bem como à informação diária e freqüente $(\mathrm{a}$ cada momento da administração) acerca do nome, via, dose, horário e propósito da medicação.

\section{Referências}

1. Arcuri EAM. Reflexões sobre a responsabilidade do enfermeiro na administração de medicamentos. Revista da Escola de Enfermagem da USP, São Paulo 1991 ago;25(2):229-37.

2. Arndt M. Nurses medication errors. Journal of Advanced Nursing, Oxford 1994 Mar;19(3):519-26.

3. Cassiani SHB. Administração de medicamentos. São Paulo: EPU; 2000. 131 p. il.

4. Zaneti ACG, Cassiani SHB. Erros na administração de medicamentos: percepção dos enfermeiros. Âmbito Hospitalar: Revista Científica para Profissionais da Saúde, São Paulo 2000;12(135):20-6.

5. Carvalho VT. Erros na administração de medicamentos: análise de relatos dos profissionais de enfermagem [dissertação de Mestrado]. Ribeirão Preto (SP): Escola de Enfermagem de Ribeirão Preto, Universidade de São Paulo, 2000. 139 f.

6. Bueno E, Cassiani SHB, Miquelin JDL. Erros na administração de medicamentos: fatores de risco e medidas empregadas. Revista Baiana de Enfermagem, Salvador (BA) 1998 abr;11(1):101-19.

7. Flynn ER, Wolf ZR, McGoldrick TB, Jablonski RA, Dean LM, McKee EP. Effect of three teaching methods on a nursing staff's knowledge of medication error risk reduction strategies. Journal of Nursing Staff Development, Philadelphia (PA) 1996 Jan/Feb;12(1):19-26.

8. Cassiani SHB. Erros na medicação: estratégias de prevenção. Revista Brasileira de Enfermagem, Brasília (DF) 2000 jul/set;53(3):424-30.

9. Telles Filho PCP, Galdeano LE, Miasso AI, Caliri MHL, Mendes IAC. Administração de medicamentos e a comunicação de conhecimento via online. Revista Brasileira de Enfermagem, Brasília (DF) 2001 out/ dez;54(4):539-47

10. Davis NM. Teaching patientes to prevent errors. American Journal of Nursing, New York 1994 May;94(5):17.

11. Resnick B, Slocum D, Moffett P. Geriatric rehabilitation: nursing focusing on urinary function and knowledge of medication. Rehabilitation Nursing, Glenview (IL) 1996 May-June;21(3):142-7.

12. Merkatz R, Couig MP. Helping America take its medicine. American Journal of Nursing, New York 1992 June;92(6):56-60.

13. Trevelian J. Talking their own Medicine. Nursing Times and Nursing Mirror, London 1988 Jan 27;84(4):28-32.

14. Cassiani SHB, Gimenes FRE, Freire CC. Avaliação da prescrição médica eletrônica em um hospital universitário. Revista Brasileira de Enfermagem, Brasília (DF) 2002 set/out;55(5):509-13. 